\title{
ON THE STABILITY OF LINEAR GANONICAL SYSTEMS WITH PERIODIC COEFFICIENTS
}

\author{
W. A. COPPEL and A. HOWE \\ (received 9 October 1964)
}

\section{Introduction}

By a linear canonical system we mean a system of linear differential equations of the form

$$
J x^{\prime}=H(t) x,
$$

where $J$ is an invertible skew-Hermitian matrix and $H(t)$ is a continuous Hermitian matrix valued function. We reserve the name Hamiltonian for real canonical systems with

$$
J=\left(\begin{array}{cc}
0 & I_{k} \\
-I_{k} & 0
\end{array}\right)
$$

where $I_{k}$ denotes the $k \times k$ unit matrix. In recent years the stability properties of Hamiltonian systems whose coefficient matrix $H(t)$ is periodic have been deeply investigated, mainly by Russian authors ([2], [3], [5], [7]). An excellent survey of the literature is given in [6]. The purpose of the present paper is to extend this theory to canonical systems. The only work which we know of in this direction is a paper by Yakubovič [9].

The plan of the paper is as follows. In $\S 2$ we give necessary and sufficient conditions for a periodic canonical system to be strongly stable. This question is discussed in the paper by Yakubovič cited above. However our treatment differs somewhat from his and the results are fundamental for what follows. In $\S 3$ we carry over to canonical systems the elegant theory of stability domains due to Gelfand and Lidskir [3]. In $\S 4$ we prove perturbation theorems analogous to those obtained by Diliberto [2] in the Hamiltonian case. The problem of extending these results to canonical systems was proposed by Krein in the discussion following Diliberto's address.

The following notation will be adopted throughout the paper. We will denote by $\mathscr{V}$ the vector space formed by all vectors $x=\left(\xi_{1}, \cdots, \xi_{n}\right)$ with complex coordinates. We will suppose that $\mathscr{V}$ is normed in some 
way and we will denote by $|x|$ the length of the vector $x$. For any $n \times n$ matrix $A$, representing a linear transformation of $\mathscr{V}$, we set

$$
|A|=\sup _{|x|=1}|A x| .
$$

The conjugate transpose of $A$ will be denoted by $A^{*}$ and the diagonal matrix with diagonal elements $\lambda_{1}, \cdots, \lambda_{n}$ will be denoted by $\left[\lambda_{1}, \cdots, \lambda_{n}\right]$.

In studying the canonical system (1) there is no loss of generality in supposing that

$$
J=i\left(\begin{array}{cc}
I_{p} & 0 \\
0 & -I_{q}
\end{array}\right),
$$

where $0 \leqq p, q$ and $p+q=n$. We will always assume that $J$ has this form, although many of our results will be independent of this assumption.

\section{Strong Stability}

For purposes of comparison with later results we first derive some well-known properties of an arbitrary homogeneous linear system

$$
x^{\prime}=A(t) x,
$$

whose coefficient matrix $A(t)$ is continuous and periodic:

$$
A(t+\omega)=A(t) .
$$

The system (3) is said to be (two-sided) stable if each solution $x(t)$ is bounded for $-\infty<t<\infty$. If $X(t)$ denotes the fundamental matrix for (3) such that $X(0)=I$ then (3) is stable if and only if $X(t)$ is bounded, since the solutions of (3) are given by $x(t)=X(t) c$ for an arbitrary constant vector $c$. Now

$$
X(t+\omega)=X(t) X(\omega),
$$

since both sides are fundamental matrices for (3) and they take the same value for $t=0$. It follows by induction that for any integer $m$

$$
X(t+m \omega)=X(t) X^{m}(\omega) .
$$

Since $X(t)$ is certainly bounded for $0 \leqq t \leqq \omega$ it will be bounded for all real $t$ if and only if the integral powers of the matrix $X(\omega)$ are bounded. The matrix $X(\omega)$ is called the monodromy matrix of the system (3).

We will say that an invertible matrix $S$ is stable if there exists a positive constant $\alpha$ such that

$$
\left|S^{m}\right| \leqq \alpha \quad(m=0, \pm 1, \pm 2, \cdots) .
$$

Then the preceding argument is summed up in 
THEOREM 1. The system (3) is stable if and only if its monodromy matrix is stable.

We must now determine under what conditions a matrix is stable. Before doing so, however, we recall some general facts about matrices.

Let $\lambda$ be an eigenvalue of the matrix $A$. The set of all vectors $x$ such that $A x=\lambda x$ is a subspace $\mathscr{V}_{\lambda}$ of the $n$-dimensional vector space $\mathscr{V}$, called the eigenspace of $A$ corresponding to the eigenvalue $\lambda$. The set of all vectors $x$ such that

$$
(A-\lambda I)^{m} x=0
$$

for some positive integer $m$ is also a subspace $\mathscr{W}_{\lambda}$, called the root space of $A$ corresponding to the eigenvalue $\lambda$. Evidently $\mathscr{V}_{\lambda} \subseteq \mathscr{W}_{\lambda}$. We will have strict inclusion, $\mathscr{V}_{\lambda} \subset \mathscr{W}_{\lambda}$, if and only if there exists a vector $x_{1}$ such that

$$
x_{2}=(A-\lambda I) x_{1} \neq 0 \text { and } A x_{2}=\lambda x_{2} .
$$

If $\lambda_{1}, \cdots, \lambda_{r}$ are the distinct eigenvalues of the matrix $A$ the whole vector space $\mathscr{V}$ is the direct sum of the corresponding root spaces:

$$
\mathscr{V}=\mathscr{W}_{1}+\cdots+\mathscr{W}_{r} .
$$

We will denote by $P_{k}$ the corresponding projection of $\mathscr{V}$ onto $\mathscr{W}_{k}$. Let $\gamma$ be a positively oriented smooth simple closed curve in the complex plane which does not pass through any eigenvalue of $A$. Then ([8])

$$
\frac{1}{2 \pi i} \int_{\gamma}(\zeta I-A)^{-1} \mathrm{~d} \zeta=\sum P_{k},
$$

where the summation on the right is over all projections $P_{k}$ whose corresponding eigenvalues $\lambda_{k}$ lie inside $\gamma$.

We will now establish necessary and sufficient conditions for a matrix to be stable.

THEOREM 2. A matrix is stable if and only if its eigenvalues all have absolute value 1 and its eigenvectors span the vector space $\mathscr{V}$.

(This result can also be formulated in the following manner: a matrix $S$ is stable if and only if there exists an invertible matrix $T$ such that $S=T D T^{-1}$, where

$$
D=\left[e^{i \theta_{1}}, \cdots, e^{i \theta_{n}}\right]
$$

is a diagonal matrix with diagonal elements of absolute value 1.)

Let $S$ be a stable matrix. Then it is invertible and so does not have zero as an eigenvalue. If $x$ is an eigenvector, belonging to the eigenvalue $\lambda \neq 0$ then $S^{m} x=\lambda^{m} x$ for all integers $m$. Since $S$ is stable it follows that $|\lambda|=1$. If the eigenvectors of $S$ did not span the entire space there would exist an eigenvalue $\lambda$ and non-zero vectors $x_{1}, x_{2}$ such that 


$$
S x_{1}=\lambda x_{1}+x_{2}, S x_{2}=\lambda x_{2} .
$$

Then for all positive integers $m$

$$
S^{m} x_{1}=\lambda^{m} x_{1}+m \lambda^{m-1} x_{2},
$$

which contradicts the assumption that $S$ is stable.

Conversely let $S$ be a matrix satisfying the two conditions of the theorem. If $\lambda_{k}(k=1, \ldots, r)$ are the distinct eigenvalues of $S$ and $\mathscr{V}_{k}$ $(k=1, \cdots, r)$ the corresponding eigenspaces then

$$
\mathscr{V}=\mathscr{V}_{1}+\cdots+\mathscr{V}_{.} .
$$

Let $P_{k}$ denote the corresponding projection of $\mathscr{V}$ onto $\mathscr{V}_{k}$. Then for any $x \in \mathscr{V}$

$$
x=P_{1} x+\cdots+P_{r} x
$$

and hence

$$
\begin{aligned}
S^{m} x & =\lambda_{1}^{m} P_{1} x+\cdots+\lambda_{r}^{m} P_{r} x, \\
\left|S^{m} x\right| & \leqq\left(\left|P_{1}\right|+\cdots+\left|P_{r}\right|\right)|x| .
\end{aligned}
$$

Thus $S$ is stable.

For autonomous linear systems we can obtain more complete results.

THEOREM 3. The autonomous linear system

$$
x^{\prime}=A x
$$

is stable if and only if the eigenvalues of the constant matrix $A$ are all pure imaginary and the corresponding eigenvectors span the vector space $\mathscr{V}$.

The proof is similar to that of the preceding theorem. If $x_{0}$ is an eigenvector of $A$ belonging to the eigenvalue $\lambda$ then

$$
x(t)=e^{t A} x_{0}=e^{\lambda t} x_{0}
$$

is a solution of (4) which is bounded for all real $t$ if and only if $\lambda$ is pure imaginary. If there existed an eigenvalue $\lambda$ and non-zero vectors $x_{1}, x_{2}$ such that

$$
A x_{1}=\lambda x_{1}+x_{2}, A x_{2}=\lambda x_{2}
$$

then

$$
x(t)=e^{t A} x_{1}=e^{\lambda t}\left(x_{1}+t x_{2}\right)
$$

would be an unbounded solution of (4).

This proves the necessity of the conditions. Conversely if the conditions are satisfied there exist $n$ linearly independent bounded solutions of the form $x(t)=e^{\lambda t} x_{0}$. 
We now restrict attention to systems (1) of canonical form. If $X(t)$ is the fundamental matrix for (1) such that $X(0)=I$ then

$$
X^{*}(t) J X(t)=J
$$

for all real $t$. In fact, differentiating,

$$
\begin{aligned}
\left(X^{*} J X\right)^{\prime} & =X^{*} J X^{\prime}+X^{\prime *} J X \\
& =X^{*} J X^{\prime}-\left(J X^{\prime}\right)^{*} X \\
& =X^{*} H X-X^{*} H X=0 .
\end{aligned}
$$

Thus $X^{*} J X$ is a constant matrix whose value can be found by setting $t=0$.

A matrix $S$ such that

$$
S^{*} J S=J
$$

is said to be J-unitary. Any $J$-unitary matrix $S$ is invertible and $S^{-1}=$ $J^{-1} S^{*} J$. It follows that if $\lambda$ is an eigenvalue of a $J$-unitary matrix $S$ then $\bar{\lambda}^{-1}$ is also. Thus the spectrum of a $J$-unitary matrix is invariant under inversion in the unit circle. The set of all $J$-unitary matrices is a group with respect to matrix multiplication. We will always denote this group by $\mathscr{G}$. If $J= \pm i I$ a $J$-unitary matrix is unitary in the ordinary sense.

If $H$ is a constant Hermitian matrix then $e^{J^{-1} H}$ is $J$-unitary, since $X(t)=e^{t^{-1} H}$ is the fundamental matrix for the autonomous system $J x^{\prime}=H x$ such that $X(0)=I$.

The system (1) is said to be strongly stable if all neighbouring canonical systems are stable, i.e. if there exists an $\varepsilon>0$ such that the system

$$
J x^{\prime}=K(t) x
$$

is stable for any continuous Hermitian matrix $K(t)$ of period $\omega$ satisfying

$$
|K(t)-H(t)| \leqq \varepsilon \text { for } 0 \leqq t \leqq \omega .
$$

We will say that a $J$-unitary matrix $S$ is strongly stable if there exists an $\varepsilon>0$ such that any $J$-unitary matrix $T$ satisfying

$$
|T-S| \leqq \varepsilon
$$

is stable. The connection between these two concepts is provided by

THEOREM 4. The system (1) is strongly stable if and only if its monodromy matrix is strongly stable.

It follows at once from Theorem 1 that the system (1) is strongly stable if its monodromy matrix $X(\omega)$ is strongly stable, since the solutions of (1) depend continuously on the coefficient matrix. Suppose on the other hand that the monodromy matrix is stable without being strongly stable. 
Then by the proof of the following Theorem 5 in any neighbourhood of $X(\omega)$ there exists a $J$-unitary matrix $T$ which has an eigenvalue of absolute value greater than 1 .

The mapping $A \rightarrow e^{A}$ maps a neighbourhood of 0 in the space of all $n \times n$ matrices topologically onto a neighbourhood of $I$ in the group of all invertible matrices $([1]$, p. 7$)$. Thus we can write

$$
X^{-1}(\omega) T=e^{A},
$$

where $|A|$ is small. Since $X^{-1}(\omega) T$ is $J$-unitary we have

$$
e^{-A}=J^{-1} e^{A^{*} J}=e^{J^{-1} A^{*} J} .
$$

Therefore, since the mapping is $1-1$,

$$
-A=J^{-1} A^{*} J \text {. }
$$

Thus we can write $A=\omega J^{-1} C$, where $C$ is Hermitian.

Now $Y(t)=X(t) e^{t J^{-1} C}$ satisfies $Y(0)=I$ and is a fundamental matrix for the system

$$
J x^{\prime}=K(t) x,
$$

where

$$
K(t)=H(t)+J X(t) J^{-1} C X^{-1}(t)
$$

is Hermitian. Moreover $Y(\omega)=T$. It is possible that $K(\omega) \neq K(0)$, but in any case

$$
|K(\omega)-K(0)| \leqq \alpha|C|,
$$

for some positive constant $\alpha$ independent of $C$. By altering $K(t)$ slightly for values of $t$ near $\omega$ we can ensure that $K(\omega)=K(0)$, while $Y(\omega)$ remains so close to $T$ that it still has an eigenvalue of absolute value greater than 1 . Thus the system (1) is not strongly stable.

We must now determine under what conditions a $J$-unitary matrix is strongly stable. We first introduce a metric into our vector space $\mathscr{V}$ by defining for any two vectors $x, y$

$$
(x, y)=i^{-1} y^{*} J x \text {. }
$$

Evidently

$$
|(x, y)| \leqq|J||x||y|
$$

Since $i^{-1} J$ is Hermitian we have $(y, x)=\overline{(x, y)}$. The real number $(x, x)$ will be called the $(J$-)norm of the vector $x$. A subspace $\mathscr{W}$ of $\mathscr{V}$ will be said to be non-negative if $(x, x) \geqq 0$ for every $x \in \mathscr{W}$ and positive if $(x, x)>0$ for every $x \neq 0 \in \mathscr{W}$. Similarly for non-positive and negative subspaces. Two vectors $x, y$ such that $(x, y)=0$ will be said to be orthogonal. If $x$ is 
orthogonal to every vector $y$ then $J x=0$ and hence $x=0$. A matrix $S$ is $J$-unitary if and only if

$$
(S x, S y)=(x, y)
$$

for all vectors $x, y$. If we set

$$
e_{1}=(1,0, \cdots, 0), e_{2}=(0,1, \cdots, 0), \cdots, e_{n}=(0,0, \cdots, 1)
$$

then by (2)

(6) $\left(e_{j}, e_{k}\right)=0$ for $j \neq k$,

$$
\left(e_{j}, e_{j}\right)=1 \text { for } 1 \leqq j \leqq p,=-1 \text { for } p<j \leqq n \text {. }
$$

Lemma 1. The eigenspaces of a stable J-unitary matrix $S$ are mutually orthogonal.

For suppose $x, y$ are eigenvectors belonging to the distinct eigenvalues $\lambda, \mu$. Then

$$
\lambda \bar{\mu}(x, y)=(S x, S y)=(x, y) .
$$

But $\lambda \bar{\mu} \neq 1$, because $\lambda$ has absolute value 1 and $\lambda \neq \mu$. Therefore $(x, y)=0$.

Since a non-zero vector cannot be orthogonal to the whole of $\mathscr{V}$ we have as a

COROLlary. An eigenvector of a stable J-unitary matrix cannot be orthogonal to the eigenspace to which it belongs.

We next prove

Lemma 2. If an eigenspace $\mathscr{W}$ of a stable $J$-unitary matrix is nonnegative then it is positive.

Suppose $x \in \mathscr{W}$ and $(x, x)=0$. We must show that $x=0$. For any vector $y \in \mathscr{W}$ and any scalar $\lambda$

$$
0 \leqq(y+\lambda x, y+\lambda x)=(y, y)+2 \mathscr{R} \lambda(x, y) .
$$

If $(x, y)$ were different from zero we could make the right side negative by suitable choice of $\lambda$. Therefore $x$ is orthogonal to $\mathscr{W}$, and so by the preceding corollary $x=0$.

THEOREM 5. A stable J-unitary matrix is strongly stable if and only if each of its eigenspaces is definite (i.e. positive or negative).

Let $S$ be a stable $J$-unitary matrix and suppose that for some eigenvalue $\lambda$ with $|\lambda|=1$ the corresponding eigenspace is not definite. Then by Lemma 2 there exist eigenvectors corresponding to the eigenvalue $\lambda$ with both positive and negative norms. Hence we can find eigenvectors $x_{1}, x_{2}$ such that

$$
\left(x_{1}, x_{1}\right)=1,\left(x_{1}, x_{2}\right)=0,\left(x_{2}, x_{2}\right)=-1 .
$$


Choose $x_{3}, \cdots, x_{n}$ orthogonal to $x_{1}$ and $x_{2}$ so that $x_{1}, \cdots, x_{n}$ form a basis for $\mathscr{V}$ and define a linear transformation $T$ by setting

$$
\begin{aligned}
& T x_{1}=\lambda\left(\cosh \alpha x_{1}+\sinh \alpha x_{2}\right) \\
& T x_{2}=\lambda\left(\sinh \alpha x_{1}+\cosh \alpha x_{2}\right) \\
& T x_{k}=S x_{k} \text { if } k>2 .
\end{aligned}
$$

It is easily verified that $\left(T x_{j}, T x_{k}\right)=\left(x_{j}, x_{k}\right)$ for all $j, k$. Thus $T$ is $J$-unitary. Moreover $x_{1}+x_{2}$ is an eigenvector of $T$ belonging to the eigenvalue $\lambda e^{\alpha}$, so that $T$ has an eigenvalue outside the unit circle for any $\alpha>0$. On the other hand $T$ is arbitrarily close to $S$ if $\alpha$ is sufficiently small. Hence $S$ is not strongly stable.

Suppose now that $S$ is a stable $J$-unitary matrix each of whose eigenspaces is positive or negative. Let $\lambda_{k}(k=1, \cdots, r)$ be the distinct eigenvalues of $S$ and let $\mathscr{V}_{k}(k=1, \cdots, r)$ be the corresponding eigenspaces, so that

$$
\mathscr{V}=\mathscr{V}_{1}+\cdots+\mathscr{V}_{r} .
$$

If $\gamma_{k}$ is the positively oriented circumference of a circle with centre $\lambda_{k}$ and radius so small that it contains no other eigenvalue of $S$ then

$$
P_{k}=\frac{1}{2 \pi i} \int_{\gamma_{k}}(\zeta I-S)^{-1} d \zeta
$$

denotes the corresponding projection of $\mathscr{V}$ onto $\mathscr{V}_{k}$. Since the eigenspaces are definite there exists a positive constant $\alpha$ such that

$$
|(x, x)| \geqq \alpha|x|^{2} \text { for } x \in \mathscr{V}_{k} \quad(k=1, \cdots, r) .
$$

If the matrix $T$ is close enough to $S$ every eigenvalue of $T$ will lie inside one of the circles $\gamma_{k}$. Then

$$
Q_{k}=\frac{1}{2 \pi i} \int_{\gamma_{k}}(\zeta I-T)^{-1} d \zeta
$$

is a projection which commutes with $T$ and $\mathscr{V}$ is the direct sum of the invariant subspaces $\mathscr{W}_{k}=Q_{k} \mathscr{V}(k=1, \cdots, r)$. For $x \in \mathscr{W}_{k}$ we have

$$
\begin{aligned}
(x, x)= & \left(Q_{k} x, Q_{k} x\right) \\
=\left(P_{k} x, P_{k} x\right) & +\left(\left(Q_{k}-P_{k}\right) x,\left(Q_{k}-P_{k}\right) x\right) \\
& +2 \mathscr{R}\left(P_{k} x,\left(Q_{k}-P_{k}\right) x\right) .
\end{aligned}
$$

If in addition $|x|=1$ then

$$
\left|P_{k} x\right| \geqq\left|Q_{k} x\right|-\left|Q_{k}-P_{k}\right||x|=1-\left|Q_{k}-P_{k}\right|
$$

and hence 


$$
|(x, x)| \geqq \alpha\left(1-\left|Q_{k}-P_{k}\right|\right)^{2}-|J|\left|Q_{k}-P_{k}\right|^{2}-2|J|\left|P_{k}\right|\left|Q_{k}-P_{k}\right| .
$$

Consequently if $T$ is sufficiently close to $S$ then

$$
|(x, x)| \geqq \frac{1}{2} \alpha|x|^{2} \text { for } x \in \mathscr{W}_{k} \quad(k=1, \cdots, r) .
$$

If $T$ is also $J$-unitary then, since $\mathscr{W}_{k}$ is invariant under $T$, we have for all $x \in \mathbb{W}_{k}$

$$
\begin{aligned}
\left|T^{m} x\right|^{2} & \leqq 2 \alpha^{-1}\left|\left(T^{m} x, T^{m} x\right)\right| \\
& =2 \alpha^{-1}|(x, x)| \leqq 2 \alpha^{-1}|J||x|^{2} .
\end{aligned}
$$

Thus there exists a positive constant $\beta$ such that

$$
\left|T^{m} x\right| \leqq \beta|x| \text { for } x \in \mathscr{W}_{k} \quad(k=1, \cdots, r) .
$$

But for any $x \in \mathscr{V}$

and hence

$$
x=Q_{1} x+\cdots+Q_{r} x
$$

$$
\left|T^{m} x\right| \leqq \beta\left(\left|Q_{1}\right|+\cdots+\left|Q_{\tau}\right|\right)|x| .
$$

Thus all $J$-unitary matrices near $S$ are stable, and $S$ is strongly stable.

We can obtain more explicit results by using the specific form (2) of the matrix $J$.

THEOREM 6. A matrix $S$ is a stable $J$-unitary matrix if and only if it can be represented in the form

$$
S=G D G^{-1},
$$

where $G$ is a J-unitary matrix and

$$
D=\left[e^{i \theta_{1}}, \cdots, e^{i \theta_{m}}\right]
$$

is a diagonal matrix whose diagonal elements have absolute value 1 .

If $S$ has the form (7) then it is stable, by Theorem 2 , and $J$-unitary because $G$ and $D$ are $J$-unitary. Conversely, let $S$ be a stable $J$-unitary matrix and let $\lambda$ be any eigenvalue of $S$, of multiplicity $r$ say. By the Corollary to Lemma $I$ we can choose an orthonormal basis $x_{1}, \cdots, x_{r}$ for the eigenspace $\mathscr{V}_{\lambda}$ so that

$$
\left(x_{i}, x_{k}\right)=0 \text { if } j \neq k,= \pm 1 \text { if } j=k \quad(j, k=1, \cdots, r) .
$$

By stringing together orthonormal bases for each of the eigenspaces we obtain, by Lemma 1 itself, an orthonormal basis $g_{1}, \cdots, g_{n}$ for the whole space. We can suppose the eigenvectors $g_{1}, \cdots, g_{n}$ ordered so that

$$
\begin{aligned}
& \left(g_{j}, g_{k}\right)=0 \text { if } j \neq k, \\
& \left(g_{j}, g_{j}\right)=1 \text { if } j \leqq p^{\prime},=-1 \text { if } j>p^{\prime} .
\end{aligned}
$$


By (6) and the law of inertia for Hermitian forms we must have $p^{\prime}=p$. Let $e^{i \theta_{j}}$ be the eigenvalue associated with the eigenvector $g_{j}(j=1, \cdots, n)$. We will say that the eigenvalues $e^{i \theta_{s}}(j=1, \cdots, p)$ are of positive type and the eigenvalues $e^{i \theta_{s}}(j=p+1, \cdots, n)$ of negative type.

If $G$ is the matrix with columns $g_{1}, \cdots, g_{n}$ then (7) holds. Moreover $G$ is $J$-unitary, since

$$
\left(G e_{j}, G e_{k}\right)=\left(g_{j}, g_{k}\right)=\left(e_{j}, e_{k}\right) .
$$

COROLlaRY. A stable J-unitary matrix $S$ is strongly stable if and only if in the representation (7)

$$
\theta_{j} \not \theta_{k}(\bmod 2 \pi) \text { for } 1 \leqq j \leqq p<k \leqq n .
$$

For this is the condition that an eigenvalue should not be simultaneously of positive and negative type, i.e. that each of the eigenspace be definite.

Finally we consider autonomous linear canonical systems.

THEOREM 7. The linear canonical system

$$
J x^{\prime}=H x,
$$

where $H$ is a constant Hermitian matrix, is stable if and only if there exists a J-unitary matrix $G$ such that

$$
F=G^{*} H G
$$

is a real diagonal matrix:

$$
F=\left[\lambda_{1}, \cdots, \lambda_{n}\right] .
$$

If (10) holds then

$$
J^{-1} H=G\left(J^{-1} F\right) G^{-1}
$$

and (9) is stable by Theorem 3. Conversely, suppose (9) is stable. Then by Theorem 3 the eigenvalues of $A=J^{-1} H$ are pure imaginary and the corresponding eigenvectors span the vector space $\mathscr{V}$. For any two vectors $x, y$ we have

$$
(A x, y)=\left(x, J^{-1} A^{*} J y\right)=-(x, A y),
$$

since $J^{-1} A^{*} J=-A$. If $x, y$ are eigenvectors corresponding to the distinct eigenvalues $\lambda, \mu$ then

$$
\lambda(x, y)=(A x, y)=-(x, A y)=-\bar{\mu}(x, y) .
$$

Since $-\bar{\mu}=\mu \neq \lambda$ it follows that $(x, y)=0$. Hence, as in the proof of Theorem 6 , we can find $n$ linearly independent eigenvectors $g_{1}, \cdots, g_{n}$ such that 


$$
\begin{aligned}
& \left(g_{j}, g_{k}\right)=0 \text { if } j \neq k, \\
& \left(g_{j}, g_{j}\right)=1 \text { if } j \leqq p,=-1 \text { if } j>p .
\end{aligned}
$$

The matrix $G$ with columns $g_{1}, \cdots, g_{n}$ is $J$-unitary and

$$
A=G\left(J^{-1} F\right) G^{-1},
$$

where $F$ is a real diagonal matrix. Substituting $A=J^{-1} H$ we obtain (10) ${ }_{1}$.

COROLlary. The autonomous canonical system (9) is strongly stable, for given $\omega>0$, if and only if in the representation (10)

$$
\lambda_{j}+\lambda_{k} \neq 0(\bmod 2 \pi / \omega) \text { for } 1 \leqq j \leqq p<k \leqq n .
$$

In fact the monodromy matrix of $(9)$ is $e^{\omega J^{-1} H}$ and

$$
G^{-1} e^{\omega J^{-1} H} G=e^{\omega J^{-1} F},
$$

Thus the monodromy matrix has the form (7) with

$$
D=\left[e^{-i \omega \lambda_{1}}, \cdots, e^{-i \omega \lambda_{n}}, e^{i \omega \lambda_{p+1}}, \cdots, e^{i \omega \lambda_{n}}\right]
$$

and the result follows from the Corollary to Theorem 6.

\section{Domains of Stability}

It follows at once from the definition of strongly stable systems that they form an open set in the space of all linear canonical systems. Like any open set this set can be represented as a union of disjoint connected (nonempty) open sets, which we will call domains of stability. Two strongly stable systems belong to the same domain of stability if and only if they can be continuously deformed into one another without ceasing to be strongly stable. Two questions immediately arise: how many stability domains are there, and how can we decide to which domain a given strongly stable system belongs?

There is a $1-1$ correspondence between canonical systems (1) with continuous periodic coefficient matrix $H(t)$ and continuously differentiable curves $X(t)(0 \leqq t \leqq \omega)$ in the $J$-unitary group $\mathscr{G}$ such that $X(0)=I$ and $X^{\prime}(\omega)=X^{\prime}(0) X(\omega)$, the correspondence being given explicitly by

$$
H(t)=J X^{\prime}(t) X^{-1}(t) .
$$

Thus we are led to ask when two such curves in $\mathscr{G}$ can be continuously deformed into one another.

We first consider a related but somewhat simpler question: when can two strongly stable $J$-unitary matrices be continuously deformed into one another without ceasing to be strongly stable? To answer this question we associate with any strongly stable $J$-unitary matrix $S$ a sequence of $p$ 
plus signs and $q$ minus signs, corresponding to the eigenvalues of $S$ as the circumference of the unit circle is described positively, starting from 1 . A plus sign is used to represent an eigenvalue of positive type, a minus sign for an eigenvalue of negative type, and multiple eigenvalues are repeated as often as their multiplicity. For example, the sequence $(+-++-)$ corresponds to the distribution of eigenvalues shown in the figure.

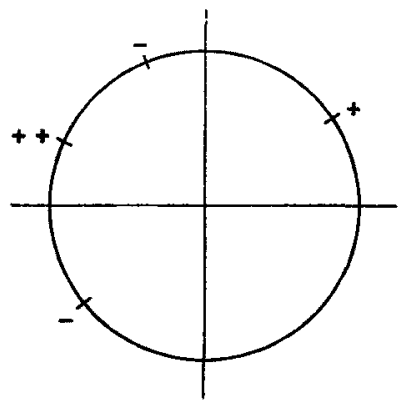

Fig. 1.

We call the signature of a strongly stable $J$-unitary matrix the corresponding sequence of + and - signs without regard to their cyclic order. Thus we identify the sequence in the example above with the sequence $(++-+-)$. The determination of the total number of different signatures ${ }^{*}$ is an interesting combinatorial problem, which was discussed by Jablonski [4]. If $p$ or $q$ is zero there is of course only one signature. If $p$ and $q$ are positive and relatively prime the total number is $(n-1) ! / p ! q !$ In the general case the total number is

$$
\frac{1}{n} \sum_{d \mid(p, q)} \frac{\varphi(d)(n / d) !}{(p / d) !(q / d) !}
$$

where $(p, q)$ is the greatest common divisor of $p$ and $q$ and $\varphi(d)$ is Euler's $\varphi$-function.

THEOREM 8. Two strongly stable J-unitary matrices can be continuously deformed into one another without ceasing to be strongly stable if and only if they have the same signature.

The necessity of the condition is immediate, since if it were not satisfied then in the course of transforming one strongly stable $J$-unitary matrix into the other an eigenvalue of positive type would have to coincide with an eigenvalue of negative type.

* This number is equal to the number of different rings of $p$ white and $q$ red beads. 
Suppose on the other hand that the two strongly stable $J$-unitary matrices $S_{1}$ and $S_{2}$ have the same signature. By Theorem 6 we can write

$$
S_{1}=G_{1} D_{1} G_{1}{ }^{-1}, \quad S_{2}=G_{2} D_{2} G_{2}{ }^{-1} .
$$

where $G_{h}$ is $J$-unitary and $D_{h}$ is diagonal $(h=1,2)$. Moreover we can suppose that the diagonal elements of $D_{1}$ have the same cyclic distribution on the unit circumference as the corresponding diagonal elements of $D_{2}$. Evidently $D_{1}$ can be continuously deformed into $D_{2}$ through diagonal matrices with diagonal elements of absolute value 1 without violating the condition (8). Then $G_{1}$ can be continuously deformed into $G_{2}$ along a curve in $\mathscr{G}$. (The connectivity of $\mathscr{G}$ follows from results established later). Throughout the whole process of deformation we remain within the set of strongly stable $J$-unitary matrices, by Theorem 6 again.

We have seen that to every strongly stable canonical system there corresponds a curve $X(t)(0 \leqq t \leqq \omega)$ in the group $\mathscr{G}$. The result which has just been established tells us when the ends $X(\omega)$ of two such curves can be continuously deformed into one another without ceasing to be the monodromy matrices of strongly stable systems. This is necessary but not sufficient for the whole curves $X(t)$ to be continuously deformable into one another without ceasing to be the fundamental matrices of strongly stable systems. To proceed further we must study the topological structure of the $J$-unitary group.

LEMMA 3. If $p$ and $q$ are both positive the J-unitary group is homeomorphic to the topological product of a torus and a simply-connected, connected topological space.

Any invertible matrix $S$ can be represented in the form $S=P U$, where $P$ is positive Hermitian and $U$ is unitary. Moreover the representation is unique and $P$ and $U$ depend continuously on $S$, since $P=\left(S S^{*}\right)^{\frac{1}{2}}$. We will show that if $S$ is $J$-unitary the factors $P$ and $U$ are also $J$-unitary. In fact, substituting $P U$ for $S$ in the relation (5):

$$
S=J^{-1}\left(S^{*}\right)^{-1} J
$$

we obtain

$$
P U=J^{-1} P^{-1} U J=\left(J^{-1} P^{-1} J\right)\left(J^{-1} U J\right) .
$$

Since by (2) the matrix $J$ is unitary the factors $J^{-1} P^{-1} J$ and $J^{-1} U J$ are respectively positive Hermitian and unitary. It follows from the uniqueness of the polar factorisation that

$$
P=J^{-1} P^{-1} J, U=J^{-1}\left(U^{*}\right)^{-1} J,
$$

which is what we wished to prove. 
Every positive Hermitian matrix $P$ can be uniquely represented in the form $P=e^{\boldsymbol{H}}$, where $H$ is Hermitian. Moreover the mapping $H \rightarrow e^{\boldsymbol{H}}$ establishes a homeomorphism between the set of all Hermitian matrices and the set of all positive Hermitian matrices $([1], p .14)$. If $P$ is also $J$-unitary then

$$
e^{H}=e^{-J^{-1} H J} .
$$

and hence, from the uniqueness of the representation,

$$
H=-J^{-1} H J \text {. }
$$

If

$$
H=\left(\begin{array}{ll}
H_{1} & H_{2} \\
H_{2}^{*} & H_{3}
\end{array}\right)
$$

is the partition of $H$ corresponding to the partition (2) of $J$ the preceding condition is equivalent to $H_{1}=H_{3}=0$. Thus the set of all positive Hermitian matrices in $\mathscr{G}$ is homeomorphic to the set of all matrices of the form

$$
\left(\begin{array}{ll}
0 & H_{2} \\
H_{2}^{*} & 0
\end{array}\right),
$$

i.e. to a Euclidean space of dimension $2 p q$.

We consider next the set of matrices which are both unitary and $J$ unitary. The unitary matrix

$$
U=\left(\begin{array}{ll}
U_{1} & U_{2} \\
U_{3} & U_{4}
\end{array}\right)
$$

will be also $J$-unitary if and only if $U=J^{-1} U J$, therefore by (2) if and only if $U_{2}=U_{3}=0$. Thus the set of all unitary matrices in $\mathscr{G}$ coincides with the set of all matrices of the form

$$
\left(\begin{array}{ll}
U_{1} & 0 \\
0 & U_{4}
\end{array}\right)
$$

where $U_{1}$ and $U_{4}$ are unitary matrices of dimension $p \times p$ and $q \times q$.

The unitary group is the topological product of a circumference and the group of unitary matrices with determinant 1 , the latter of which is a connected and simply-connected topological group ([1], pp. 37, 60-1). Since the topological product of two connected and simply-connected spaces is again connected and simply-connected it follows that the group of $J$ unitary matrices is homeomorphic to the product of two circumferences, ${ }^{*}$

* Only one circumference is present if $p$ or $q$ is zero. 
i.e. a torus, and a simply-connected connected space. This completes the proof of the lemma.

When $S=S(t)(0 \leqq t \leqq \omega)$ describes a closed curve in $\mathscr{G}$ its projections will describe the two circumferences a certain integral number of times, say $n_{+}$and $n_{-}$times. We will call $n_{+}$and $n_{-}$the $i n d i c e s$ of the closed curve $S(t)$. Two closed curves in $\mathscr{G}$ are homotopic if and only if they have the same positive and negative indices.

For any J-unitary matrix

$$
S=\left(\begin{array}{ll}
S_{1} & S_{2} \\
S_{3} & S_{4}
\end{array}\right)
$$

We define

$$
\arg _{+} S=\arg \operatorname{det} S_{1}, \arg S=\arg \operatorname{det} S_{4} .
$$

These arguments are many-valued functions of $S$, different branches differing by multiples of $2 \pi$. However if $S(t)(0 \leqq t \leqq \omega)$ is any curve in $\mathscr{G}$ then

$$
\left.\arg _{+} S(t)\right|_{0} ^{\omega}=\arg _{+} S(\omega)-\arg _{+} S(0)
$$

and

$$
\left.\arg S(t)\right|_{\mathbf{0}} ^{\omega}=\arg _{-} S(\omega) \text { arg_ }_{-} S(0)
$$

are uniquely determined real numbers when the argument of $S(\omega)$ is obtained from that of $S(0)$ by continuous variation along the curve $S(t)$.

If $S=P U$ is the polar factorisation of $S$ then $S_{1}=P_{1} U_{1}$ and $S_{4}=P_{4} U_{4}$, where $P_{1}$ and $P_{4}$ are sections of the positive Hermitian matrix $P$ and hence have positive determinants. Consequently the indices of any closed curve $S(t) \quad(0 \leqq t \leqq \omega)$ may be calculated from the formulae

$$
n_{+}=\left.\frac{1}{2 \pi} \arg _{+} S(t)\right|_{0} ^{\omega}, n_{-}=\left.\frac{1}{2 \pi} \arg S(t)\right|_{0} ^{\omega} .
$$

Consider now the set $\mathscr{D}=\mathscr{D}(\sigma)$ of all strongly stable $J$-unitary matrices with given signature $\sigma$. By Theorem $8 \mathscr{D}$ is a domain (= connected open set) in the group $\mathscr{G}$ of all $J$-unitary matrices. We propose to study the possible indices of closed curves in $\mathscr{D}$.

Theorem 9. Suppose $p$ and $q$ are both positive. Then the indices $n_{+}$,

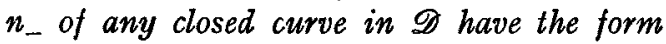

$$
n_{+}=h p, \quad n_{-}=h q,
$$

for some integer $h$. Moreover for any integer $h$ there exists a closed curve in $\mathscr{D}$ with these indices. 
In fact let $S(t)(0 \leqq t \leqq 1)$ be a closed curve in $\mathscr{D}$ and for each $t$ let

$$
S(t)=C(t) D(t) G^{-1}(t)
$$

be a representation of the form (7). It may be shown that $G(t)$ and $D(t)$ can be chosen to depend continuously on $t$ and so as to satisfy the conditions

$$
D(0)=D(1), G(0)=G(1) \text {. }
$$

Since each eigenvalue $e^{i \theta_{j}(t)}$ of $S(t)$ returns to its original value as $t$ increases from 0 to 1 then $\theta_{j}(t)$ must change by some multiple of $2 \pi$. Moreover $\theta_{j}(t)$ $(j \leqq p)$ must change by the same multiple of $2 \pi$ as $\theta_{k}(t)(k>p)$, since otherwise the condition (8) would be violated at some point of the curve. Hence all $\theta_{1}(t)(j=1, \cdots, n)$ change by the same amount $2 h \pi$. It follows from (12) that the indices of the closed curve $D(t)(0 \leqq t \leqq 1)$ are

$$
n_{+}=h p, n_{-}=h q \text {. }
$$

Let $m_{+}, m_{-}$be the indices of the closed curve $G(t)(0 \leqq t \leqq 1)$ and set

$$
\alpha=-2 \pi m_{+}, \beta=-2 \pi m_{-} .
$$

Since the diagonal matrix

$$
F(t)=\left[e^{i t \alpha}, 1, \cdots, 1, e^{i t \beta}\right]
$$

commutes with $D(t)$ we can replace $G(t)$ by $G(t) F(t)$. But

$$
\begin{aligned}
& \left.\arg _{+} G(t) F(t)\right|_{0} ^{1}=\left.\arg _{+} G(t)\right|_{0} ^{\mathbf{1}}+\alpha, \\
& \arg -\left.G(t) F(t)\right|_{0} ^{1}=\left.\arg G(t)\right|_{0} ^{\mathbf{1}}+\beta,
\end{aligned}
$$

and hence the indices of the closed curve $G(t) F(t)$ are both zero. Thus the closed curve $G(t) F(t)$ can be continuously deformed into a point within $\mathscr{G}$ and we may take this point to be the unit matrix. This shows that the closed curve $S(t)$ is homotopic in $\mathscr{D}$ to the closed curve $D(t)$. It therefore has the same indices, which proves the first part of the theorem.

The second part is trivial, since if

$$
D_{0}=\left[e^{i \theta_{1}}, \cdots, e^{i \theta_{n}}\right]
$$

is a diagonal matrix in $\mathscr{D}$ then

$$
D(t)=\left[e^{i\left(\theta_{1}+2 h \pi t\right)}, \cdots, e^{i\left(\theta_{n}+2 h \pi t\right)}\right] \quad(0 \leqq t \leqq 1)
$$

is a closed curve in $\mathscr{D}$ with indiced $n_{+}=h p, n_{-}=h q$.

Let $\mathscr{C}=\mathscr{C}(\sigma)$ denote the set of all curves $X(t)(0 \leqq t \leqq \omega)$ in $\mathscr{G}$ which start from the unit matrix and end in some point of $\mathscr{D}$. We divide $\mathscr{C}$ into subsets $\mathscr{C}_{j, m}(0 \leqq j<p ; m=0, \pm 1, \cdots)$ in the following way. 
Let $X_{0}(t)(0 \leqq t \leqq \omega)$ be a fixed curve in $\mathscr{C}$ and let $S_{0}=X_{0}(\omega)$. Let $X_{1}(t)(0 \leqq t \leqq \omega)$ be any other curve in $\mathscr{C}$ ending in the point $S_{1}=X_{1}(\omega)$. We join $S_{0}$ to $S_{1}$ by a curve $S_{1}(t)(0 \leqq t \leqq 1)$ in $\mathscr{D}$, so that

$$
S_{1}(0)=S_{0}, S_{1}(1)=S_{1} \text {. }
$$

Then

$$
\begin{aligned}
& \left.\arg _{+} X_{0}(t)\right|_{0} ^{\omega}+\left.\arg _{+} S_{1}(t)\right|_{0} ^{1}=\left.\arg _{+} X_{1}(t)\right|_{0} ^{\omega}+2 j \pi, \\
& \left.\arg X_{0}(t)\right|_{0} ^{\omega}+\left.\arg _{-} S_{1}(t)\right|_{0} ^{1}=\left.\arg X_{1}(t)\right|_{0} ^{\omega}+2 m \pi,
\end{aligned}
$$

for some integers $j, m$. It follows from Theorem 9 that by suitable choice of the curve $S_{1}(t)$ we can suppose $0 \leqq j<p$. With this restriction on $j$ the integers $j, m$ are uniquely determined by the curve $X_{1}(t)$ and we put $X_{1}(t)$ in the corresponding set $\mathscr{C}_{j, m}$.

We will now show that two curves $X_{1}(t), X_{2}(t)$ in $\mathscr{C}$ can be continuously deformed into one another without their endpoints leaving $\mathscr{D}$ if and only if they belong to the same subset $\mathscr{C}_{j, m}$. For let

$$
\begin{aligned}
& \left.\arg _{+} X_{0}(t)\right|_{0} ^{\infty}+\left.\arg _{+} S_{2}(t)\right|_{0} ^{1}=\left.\arg _{+} X_{2}(t)\right|_{0} ^{\omega}+2 j^{\prime} \pi, \\
& \left.\arg X_{0}(t)\right|_{0} ^{\omega}+\left.\arg _{-} S_{2}(t)\right|_{0} ^{1}=\left.\arg X_{2}(t)\right|_{0} ^{\omega}+2 m^{\prime} \pi,
\end{aligned}
$$

be the corresponding equations for $X_{2}(t)$, where $S_{2}(t)$ is a curve in $\mathscr{D}$ joining $S_{0}$ to $S_{2}=X_{2}(\omega)$. Then by subtraction we get

$$
\begin{aligned}
& \left.\arg _{+} X_{1}(t)\right|_{0} ^{\omega}+\left.\arg _{+} S(t)\right|_{0} ^{1}=\left.\arg _{+} X_{2}(t)\right|_{0} ^{\omega}+2\left(j^{\prime}-j\right) \pi, \\
& \left.\arg _{-} X_{1}(t)\right|_{0} ^{\omega}+\left.\arg _{-} S(t)\right|_{0} ^{1}=\left.\arg _{-} X_{2}(t)\right|_{0} ^{\omega}+2\left(m^{\prime}-m\right) \pi,
\end{aligned}
$$

where $S(t)$ is a curve in $\mathscr{D}$ joining $S_{1}$ to $S_{2}$. But $X_{1}(t)$ can be continuously deformed into $X_{2}(t)$ without its endpoint leaving $\mathscr{D}$ if and only if there exists a curve $R(t)$ in $\mathscr{D}$ joining $S_{1}$ to $S_{2}$ such that

$$
\begin{aligned}
& \left.\arg _{+} X_{1}(t)\right|_{0} ^{\omega}+\left.\arg _{+} R(t)\right|_{0} ^{1}=\left.\arg _{+} X_{2}(t)\right|_{0} ^{\omega}, \\
& \left.\arg X_{1}(t)\right|_{0} ^{\omega}+\left.\arg _{-} R(t)\right|_{0} ^{1}=\left.\arg _{-} X_{2}(t)\right|_{0} ^{\omega} .
\end{aligned}
$$

Comparing these equations with the previous ones we see from Theorem 9 that such a curve exists if and only if

$$
j^{\prime}=j+h p, m^{\prime}=m+h q
$$

for some integer $h$. On account of the restrictions on $j, j^{\prime}$ this is equivalent to $j^{\prime}=j, m^{\prime}=m$.

We show next that none of the sets $\mathscr{C}_{i, m}$ is empty. Let

$$
S_{0}=\left[e^{i \theta_{1}}, \cdots, e^{i \theta_{n}}\right]
$$

be a diagonal matrix in $\mathscr{D}$ and let

$$
X_{0}(t)=\left[e^{i t \theta_{1} / \omega}, \cdots, e^{i t \theta_{n} / \omega}\right] \quad(0 \leqq t \leqq \omega) .
$$


Evidently

$$
\left.\arg _{+} X_{0}(t)\right|_{0} ^{\infty}=\sum_{j=1}^{p} \theta_{j},\left.\arg X_{0}(t)\right|_{0} ^{\omega}=\sum_{k=p+1}^{n} \theta_{k} .
$$

For the curve $X_{1}(t)$ we take

$$
X_{1}(t)=X_{0}(t)\left[e^{i t \alpha}, 1, \cdots, 1, e^{i t \beta}\right],
$$

where $\alpha=-2 j \pi / \omega(0 \leqq j \leqq p)$ and $\beta=-2 m \pi / \omega$. This curve has the same endpoint $S_{0}$ and

$$
\left.\arg _{+} X_{1}(t)\right|_{0} ^{\omega}=\sum_{j=1}^{D} \theta_{j}-2 j \pi,\left.\arg _{-} X_{1}(t)\right|_{0} ^{\omega}=\sum_{k=p+1}^{n} \theta_{k}-2 m \pi .
$$

Thus $X_{1}(t) \in \mathscr{C}_{j, m}$.

Finally we return from curves in $\mathscr{G}$ to linear canonical systems. Let $X_{1}(t), X_{2}(t) \quad(0 \leqq t \leqq \omega)$ be the fundamental matrices for two strongly stable systems. We suppose that the monodromy matrices $X_{1}(\omega), X_{2}(\omega)$ have the same signature $\sigma$, i.e. they belong to the same domain $\mathscr{D}=\mathscr{D}(\sigma)$, and moreover that $X_{1}(t), X_{2}(t)$ belong to the same set $\mathscr{C}_{j, m}$. Then $X_{1}(t)$ can be continuously deformed into $X_{2}(t)$ in $\mathscr{G}$ without its endpoint leaving $\mathscr{D}$. It is not difficult to see that by modifying the deformation slightly we can arrange that every intermediate curve $X(t)$ is a fundamental matrix for some linear canonical system, i.e. it is continuously differentiable and satisfies the relation $X^{\prime}(\omega)=X^{\prime}(0) X(\omega)$. Consequently we can sum up the whole of the preceding argument in

THEOREM 10. If $p$ and $q$ are both positive the stability domains of the linear canonical system (1) are characterised by a signature $\sigma^{*}$, an integer $j$ such that $0 \leqq j<p$, and an arbitrary integer $m$.

Moreover, in principle at any rate we have a means of determining to which stability domain a given strongly stable system belongs. The above result requires modification if $p$ or $q$ is zero. In this case there is only one signature $\sigma$ and the domain $\mathscr{D}$ consists of all unitary matrices. It is easily seen that there exist closed curves in $\mathscr{D}$ with arbitrary index, from which it follows that there is just one domain of stability.

\section{Perturbation Theory}

Suppose we have a linear canonical system

$$
J x^{\prime}=H(t, \varepsilon) x
$$

* That is, a sequence of $p$ plus and $q$ minus signs, without regard to their cyclic order. 
depending analytically on a parameter $\varepsilon$ and suppose the unperturbed system

$$
J x^{\prime}=H(t, 0) x
$$

is strongly stable. Then the system (13) will be stable for all small $\varepsilon$. It follows from Floquet's theorem and from Theorem 7 that for each such $\varepsilon$ there exists a $J$-unitary matrix $S(t, \varepsilon)$, which is continuously differentiable in $t$ with period $\omega$, such that the change of variables $x=S(t, \varepsilon) y$ transforms (13) into an autonomous system

$$
J y^{\prime}=F(\varepsilon) y,
$$

where $F(\varepsilon)$ is a real diagonal matrix. However these results tell us nothing about the dependence of $F$ on $\varepsilon$. The object of the present section is to show that $S$ can be chosen to be analytic in $\varepsilon$, so that $F$ is also analytic in $\varepsilon$.

Let $\mathscr{H}$ denote the set of all $n \times n$ Hermitian matrices. Corresponding to the partition (2) of $J$ we can write for any matrix $C \in \mathscr{H}$

$$
C=\left(\begin{array}{cc}
H_{1} & S \\
S^{*} & H_{2}
\end{array}\right) .
$$

where $H_{1}, H_{2}$ are Hermitian matrices of dimension $p \times p, q \times q$ respectively and $S$ is some $p \times q$ matrix. A matrix $A \in \mathscr{H}$ commutes with $J$, i.e. $J^{-1} A$ is skew-Hermitian, if and only if $S=0$. A matrix $B \in \mathscr{H}$ anticommutes with $J$, i.e. $J^{-1} B$ is Hermitian, if and only if $H_{1}=H_{2}=0$.

We will denote by $\mathscr{H}_{-}$the set of all Hermitian matrices of the form

$$
\left(\begin{array}{ll}
H_{1} & 0 \\
0 & H_{2}
\end{array}\right)
$$

and by $\mathscr{H}_{+}$the set of all Hermitian matrices of the form

$$
\left(\begin{array}{ll}
0 & S \\
S^{*} & 0
\end{array}\right)
$$

Evidently $\mathscr{H}_{-}$and $\mathscr{H}_{+}$are subspaces of the real vector space $\mathscr{H}$. Moreover any $C \in \mathscr{H}$ can be uniquely represented in the form

$$
C=A+B \text {, }
$$

where $A \in \mathscr{H}_{-}$and $B \in \mathscr{H}_{+}$. It is easily verified that if $A \in \mathscr{H}_{-}$then $\exp \left(J^{-1} A\right)$ is unitary and if $B \in \mathscr{H}_{+}$then $\exp \left(J^{-1} B\right)$ is Hermitian (cf. the proof of Lemma 3).

Let

$$
F=\left(\begin{array}{ll}
F_{1} & 0 \\
0 & F_{2}
\end{array}\right)
$$


where

$$
F_{1}=\left[\lambda_{1}, \cdots, \lambda_{p}\right], F_{2}=\left[\mu_{1}, \cdots, \mu_{q}\right]
$$

are real diagonal matrices such that

$$
\lambda_{j}+\mu_{k} \neq 0(j=1, \cdots, p ; k=1, \cdots, q) .
$$

The matrix $F$ will be fixed throughout the argument which follows.

LEMMA 4. If $H(\varepsilon) \in \mathscr{H}$ is analytic in $\varepsilon$ at $\varepsilon=0$ and $H(0)=F$ there exist unique matrices $A(\varepsilon) \in \mathscr{H}_{-}$and $B(\varepsilon) \in \mathscr{H}_{+}$analytic in $\varepsilon$ at $\varepsilon=0$ such that

$$
A(0)=F, B(0)=0
$$

and

$$
\exp \left[J^{-1} B(\varepsilon)\right]^{*} H(\varepsilon) \exp \left[J^{-1} B(\varepsilon)\right]=A(\varepsilon) .
$$

We define a mapping $f$ of $\mathscr{H}$ into itself in the following way:

for

$$
C=A+B\left(A \in \mathscr{H}_{-}, B \in \mathscr{H}_{+}\right)
$$

we set

$$
f(C)=T^{*} A T, \text { where } T=\exp \left(J^{-1} B\right) .
$$

Thus $f(F)=F$ since in this case $A=F, B=0$. The relation (15) is equivalent to $H(\varepsilon)=f[A(\varepsilon)-B(\varepsilon)]$.

Since the non-zero elements of $A$ and $B$ are equal to the corresponding elements of $C$ and since $B \in \mathscr{H}_{+}$implies $T^{*}=T$ the elements of $f(C)$ are analytic functions of the elements of $C$. Hence, by the implicit function theorem for analytic mappings, it is sufficient to show that the Jacobian matrix $f_{x}$ is invertible at $F$.

For any $C \in \mathscr{H}$ the function

$$
\varphi(t)=f(F+t C)
$$

has the derivative $\varphi^{\prime}(0)=f_{x}(F) C$. The matrix $f_{x}(F)$ will be invertible if $\varphi^{\prime}(0)=0$ implies $C=0$. But if $C=A+B$, where $A \in \mathscr{H}_{-}$and $B \in \mathscr{H}_{+}$, then

$$
\varphi(t)=e^{t J^{-1} B}(F+t A) e^{t J^{-1} B}
$$

and hence

$$
\varphi^{\prime}(0)=J^{-1} B F+A+F J^{-1} B .
$$

Thus we need only show that

$$
L(B)=J^{-1} B F+F J^{-1} B
$$

maps $\mathscr{H}_{+}$into itself and $L(B)=0$ implies $B=0$. Suppose 


$$
B=\left(\begin{array}{ll}
0 & S \\
S^{*} & 0
\end{array}\right)
$$

Then

$$
L(B)=i\left(\begin{array}{lr}
0 & -S F_{2}-F_{1} S \\
S^{*} F_{1}+F_{2} S^{*} & 0
\end{array}\right) \in \mathscr{H}_{+}
$$

and $L(B)=0$ only if $S F_{2}+F_{1} S=0$. But if $S=\left(s_{j k}\right)$ then

$$
S F_{2}+F_{1} S=\left(\left(\lambda_{j}+\mu_{k}\right) s_{j k}\right) \text {. }
$$

Therefore, by (14), $S=0$ and $B=0$. This completes the proof.

By permutations of their diagonal elements, which is equivalent to replacing $F$ by $T^{*} F T$ for a particular $J$-unitary matrix $T$, we can write $F_{1}$ and $F_{2}$ as block diagonal matrices:

$$
\begin{aligned}
& F_{1}=\left[\lambda_{1} I_{p_{1}}, \cdots, \lambda_{g} I_{p_{z}}\right], \\
& F_{2}=\left[\mu_{1} I_{q_{1}}, \cdots, \mu_{t} I_{q_{t}}\right],
\end{aligned}
$$

where $\lambda_{j} \neq \lambda_{j}$, if $j \neq j^{\prime}, \mu_{k} \neq \mu_{k}$ if $k \neq k^{\prime}$ and $\lambda_{j}+\mu_{k} \neq 0(j=1, \cdots, s$; $k=1, \cdots, t)$. Corresponding to the partitions

$$
p=p_{1}+\cdots+p_{s}, q=q_{1}+\cdots+q_{t}
$$

we define $\mathscr{H}_{-}(I)$ to be set of all matrices $A \in \mathscr{H}_{-}$of the block diagonal form

$$
A=\left[H_{1}, \cdots, H_{s}, K_{1}, \cdots, K_{t}\right] \text {, }
$$

where $H_{j}$ and $K_{k}$ are Hermitian, and $\mathscr{H}_{+}\left(\Pi^{*}\right)$ to be the set of all matrices $B \in \mathscr{H}_{-}$of the form

$$
B=\left(\begin{array}{cccccc}
0 & S_{12} & S_{1 s} & & \\
& \cdots & & & 0 \\
S_{1 s}^{*} & \mathrm{~S}_{2 s}^{*} & 0 & & & \\
& & & 0 & T_{12} & T_{1 t} \\
& 0 & & \cdots & \\
& & & T_{1 t}^{*} & T_{2 t}^{*} & 0
\end{array}\right) .
$$

Evidently $\mathscr{H}_{-}(\Pi)$ and $\mathscr{H}_{-}\left(\Pi^{*}\right)$ are subspaces of $\mathscr{H}_{-}$and every $C \in \mathscr{H}_{-}$ can be uniquely expressed in the form

$$
C=A+B,
$$

where $A \in \mathscr{H}_{-}(\Pi)$ and $B \in \mathscr{H}_{-}\left(\Pi^{*}\right)$.

LEMMA 5. If $H(\varepsilon) \in \mathscr{H}_{-}$is analytic in $\varepsilon$ at $\varepsilon=0$ then there exist unique matrices $A(\varepsilon) \in \mathscr{H}_{-}(\Pi)$ and $B(\varepsilon) \in \mathscr{H}_{-}\left(\Pi^{*}\right)$ analytic in $\varepsilon$ at $\varepsilon=0$ such that 


$$
A(0)=F, B(0)=0
$$

and

$$
\exp \left[J^{-1} B(\varepsilon)\right]^{*} H(\varepsilon) \exp \left[J^{-1} B(\varepsilon)\right]=A(\varepsilon) .
$$

The proof is similar to that of the previous lemma. We define a mapping $f$ of $\mathscr{H}_{-}$into itself by setting for

$$
\begin{aligned}
& C=A+B\left(A \in \mathscr{H}_{-}(\Pi), B \in \mathscr{H}_{-}\left(I^{*}\right)\right) \\
& f(C)=T^{*} A T, \text { where } T=\exp \left(J^{-1} B\right) .
\end{aligned}
$$

Obviously $f(C) \in \mathscr{H}$. A matrix in $\mathscr{H}$ belongs to $\mathscr{H}_{-}$if and only if it commutes with $J$. Thus $J$ commutes with $A$ and $B$ and hence with $f(C)$, which proves that $f(C) \in \mathscr{H}_{-}$.

Evidently $f(F)=F$. Since the non-zero elements of $A$ and $B$ are equal to the corresponding elements of $C$ and since $B \in \mathscr{H}_{-}$implies $T^{*}=T^{-1}$ the elements of $f(C)$ are analytic functions of the elements of $C$. We need only show that the Jacobian matrix of the mapping $f$ is invertible at $F$.

For any

$$
C=A+B\left(A \in \mathscr{H}_{-}(\Pi), B \in \mathscr{H}_{-}\left(\Pi^{*}\right)\right)
$$

put

$$
\varphi(t)=f(F+t C)=e^{-t^{-1} B}(F+t A) e^{t J^{-1} B} .
$$

Then

$$
\varphi^{\prime}(0)=-J^{-1} B F+A+F J^{-1} B .
$$

It is sufficient to prove that

$$
L(B)=F J^{-1} B-J^{-1} B F
$$

maps $\mathscr{H}_{-}\left(\Pi^{*}\right)$ into itself and $L(B)=0$ implies $B=0$. But it is easily verified that if $B$ has the form (16) then $L(B)$ has the same form with $S_{j k}$ replaced by $-i\left(\lambda_{j}-\lambda_{k}\right) S_{j k}$ and $T_{j k}$ replaced by $i\left(\mu_{j}-\mu_{k}\right) T_{j k}$. This shows at once that $L(B)=0$ only if $B=0$.

The following theorem establishes the possibility of reducing autonomous canonical systems to diagonal form.

THEOREM 11. If the Hermitian matrix $H(\varepsilon)$ is analytic in $\varepsilon$ at $\varepsilon=0$ and $H(0)=F$ then there exists a J-unitary matrix $T(\varepsilon)$ analytic in $\varepsilon$ at $\varepsilon=0$ such that $T(0)=I$ and

$$
F(\varepsilon)=T^{*}(\varepsilon) H(\varepsilon) T(\varepsilon)
$$

is a real diagonal matrix.

By Lemma 4 there exists a $J$-unitary matrix $T_{1}(\varepsilon)=\exp \left[J^{-1} B_{1}(\varepsilon)\right]$, where $B_{1}(\varepsilon) \in \mathscr{H}_{+}$is analytic in $\varepsilon$ at $\varepsilon=0$ and $B_{1}(0)=0$, such that 


$$
T_{1}^{*}(\varepsilon) H(\varepsilon) T_{1}(\varepsilon)=H_{1}(\varepsilon) \in \mathscr{H}_{-}
$$

and $H_{1}(0)=F$. Then by Lemma 5 there exists a $J$-unitary matrix $\left.T_{2}(\varepsilon)=\exp []^{-1} B_{2}(\varepsilon)\right]$, where $B_{2}(\varepsilon) \in \mathscr{H}_{-}\left(\Pi^{*}\right)$ is analytic in $\varepsilon$ at $\varepsilon=0$ and $B_{2}(0)=0$, such that

$$
T_{2}^{*}(\varepsilon) H_{1}(\varepsilon) T_{2}(\varepsilon)=H_{2}(\varepsilon) \in \mathscr{H}_{-}(\Pi)
$$

and $H_{2}(0)=F$. Since we can treat the diagonal blocks of $H_{2}(\varepsilon)$ separately this reduces the theorem to the case in which $J= \pm i I$ and $F=\lambda I$. We suppose from now on that $J$ and $F$ have these forms.

Let

$$
H(\varepsilon)=F+\sum_{m=1}^{\infty} \varepsilon^{m} H_{m}
$$

be the power series expansion of $H(\varepsilon)$ which converges in some neighbourhood of $\varepsilon=0$. If there exist scalars $\rho_{m}$ such that $H_{m}=\rho_{m} I$ for all $m \geqq 1$ (e.g. if $n=1$ ) then

$$
H(\varepsilon)=\left(\lambda+\sum_{m=1}^{\infty} \varepsilon^{m} \rho_{m}\right) I
$$

and no further reduction is necessary. Otherwise there exists a least positive integer $r$ such that $H_{r} \neq \rho I$ for all real $\rho$. Then

$$
H(\varepsilon)=\left(\lambda+\varepsilon \rho_{1}+\cdots+\varepsilon^{r-1} \rho_{r-1}\right) I+\varepsilon^{r} H_{1}(\varepsilon),
$$

where

$$
H_{1}(\varepsilon)=\sum_{m=0}^{\infty} \varepsilon^{m} H_{m+r}
$$

Since the Hermitian matrix $H_{r}$ is not a multiple of the unit matrix there exists a unitary matrix $U_{1}$ such that

$$
U_{1}^{*} H_{r} U_{1}=\left[\sigma_{1} I_{n_{1}}, \cdots, \sigma_{s} I_{n_{s}}\right]
$$

where the $\sigma_{j}(j=1, \ldots, s)$ are distinct real numbers and $s>1$. Let

$$
H_{2}(\varepsilon)=U_{1}^{*} H_{1}(\varepsilon) U_{1} .
$$

Then by Lemma 5 , corresponding to the partition $\Pi$ :

$$
n=n_{1}+\cdots+n_{s},
$$

there exists a unitary matrix $U_{2}(\varepsilon)=\exp [ \pm i B(\varepsilon)]$, where $B(\varepsilon) \in \mathscr{H}\left(\Pi^{*}\right)$ is analytic in $\varepsilon$ at $\varepsilon=0$ and $B(0)=0$, such that

$$
U_{2}^{*}(\varepsilon) H_{2}(\varepsilon) U_{2}(\varepsilon)=H_{3}(\varepsilon) \in \mathscr{H}(\Pi) .
$$

It follows that

$$
U_{2}^{*}(\varepsilon) U_{1}^{*} H(\varepsilon) U_{1} U_{2}(\varepsilon)=\left(\lambda+\varepsilon \rho_{1}+\cdots+\varepsilon^{r-1} \rho_{r-1}\right) I+\varepsilon^{r} H_{3}(\varepsilon) \in \mathscr{H}(\Pi) .
$$


Since $s>1$ this reduces the theorem to matrices of lower order. By repeating the process at most $n-1$ times we obtain the theorem.

In order to carry over this result to non-autonomous systems we need a form of Floquet's theorem for canonical systems depending on a parameter. We first prove

LEMMA 6. If the J-unitary matrix $S(\varepsilon)$ is analytic in $\varepsilon$ at $\varepsilon=0$ then there exists a matrix $A(\varepsilon)$ analytic in $\varepsilon$ at $\varepsilon=0$ such that

$$
J^{-1} A^{*}(\varepsilon) J=-A(\varepsilon)
$$

and

$$
S(\varepsilon)=e^{A(\varepsilon)} .
$$

PROOF (cf. [10]). Let $\lambda_{1}, \cdots, \lambda_{r}$ be the distinct eigenvalues of $S(0)$. We surround each eigenvalue $\lambda_{j}$ by a positively oriented simple closed curve $\gamma_{j}$ so small that it does not contain the origin or any other eigenvalue. Moreover we may suppose that if $\lambda_{k}$ is obtained from $\lambda_{j}$ by inversion in the unit circle then $\gamma_{k}$ is obtained similarly from $\gamma_{j}$.

By the theory of functions of a matrix ([8])

$$
A(\varepsilon)=\frac{1}{2 \pi i} \sum_{j=1}^{r} \int_{\gamma_{s}}[\zeta I-S(\varepsilon)]^{-1} \ln \zeta d \zeta
$$

has the property (18). Moreover it is clearly analytic in $\varepsilon$ at $\varepsilon=0$. We will show that it also has the property (17). In fact

$$
J^{-1} A^{*}(\varepsilon) J=\frac{1}{2 \pi i} \sum_{j=1}^{r} \int_{\gamma_{j}}\left[\bar{\zeta} I-J^{-1} S^{*}(\varepsilon) J\right]^{-1} \ln \bar{\zeta} d \bar{\zeta} .
$$

If we denote by $\tilde{\gamma}_{j}$ the reflection of $\gamma_{j}$ in the real axis, also described positively, then

$$
J^{-1} A^{*}(\varepsilon) J=\frac{1}{2 \pi i} \sum_{j=1}^{r} \int_{\gamma_{j}}\left[\zeta I-S^{-1}(\varepsilon)\right]^{-1} \ln \zeta d \zeta
$$

or, putting $\zeta=\xi^{-1}$ and letting $\gamma_{j}^{\prime}$ denote the inverse of $\gamma_{j}$ in the unit circle,

$$
=\frac{1}{2 \pi i} \sum_{j=1}^{r} \int_{\gamma_{j}}\left[\xi^{-1} I-S^{-1}(\varepsilon)\right]^{-1} \xi^{-2} \ln \xi d \xi .
$$

Since the spectrum of the $J$-unitary matrix $S(0)$ is invariant under inversion in the unit circle the paths $\gamma_{j}^{\prime}$ are just a permutation of the paths $\gamma_{j}$. Also

$$
\xi^{-2}\left[\xi^{-1} I-S^{-1}\right]^{-1}=\xi^{-1} I-(\xi I-S)^{-1} .
$$

Since the origin lies outside each of the paths $\gamma_{j}$ it follows that 


$$
\begin{aligned}
J^{-1} A^{*}(\varepsilon) J & =-\frac{1}{2 \pi i} \sum_{j=1}^{r} \int_{\gamma_{j}}[\xi I-S(\varepsilon)]^{-1} \ln \xi d \xi \\
& =-A(\varepsilon) .
\end{aligned}
$$

THEOREM 12. Let the Hermitian matrix $H(t, \varepsilon)$ be analytic in $\varepsilon$ at $\varepsilon=0$ and continuous in $t$ with period $\omega$. Then the fundamental matrix $X(t, \varepsilon)$ of the system

$$
J x^{\prime}=H(t, \varepsilon) x
$$

can be represented in the form

$$
X(t, \varepsilon)=P(t, \varepsilon) e^{t^{-1} K(\varepsilon)}
$$

where

(i) $P(t, \varepsilon)$ and $K(\varepsilon)$ are analytic in $\varepsilon$ at $\varepsilon=0$,

(ii) $P(t, \varepsilon)$ is continuously differentiable and has period $\omega$ in $t$,

(iii) $P(t, \varepsilon)$ is J-unitary and $K(\varepsilon)$ is Hermitian.

The monodromy matrix $S(\varepsilon)=X(\omega, \varepsilon)$ is $J$-unitary and analytic in $\varepsilon$ at $\varepsilon=0$. If $A(\varepsilon)$ satisfies the conditions of Lemma 6 we can write $A(\varepsilon)=\omega J^{-1} K(\varepsilon)$, where $K(\varepsilon)$ is Hermitian and analytic in $\varepsilon$ at $\varepsilon=0$. Put

$$
P(t, \varepsilon)=X(t, \varepsilon) e^{-t J^{-1} K(\varepsilon)} .
$$

Then $P$ is $J$-unitary, because both factors are $J$-unitary, and

$$
\begin{aligned}
P(t+\omega, \varepsilon) & =X(t, \varepsilon) X(\omega, \varepsilon) e^{-A(\varepsilon)} e^{-t J^{-1} K(\varepsilon)} \\
& =X(t, \varepsilon) e^{-t J^{-1} K(\varepsilon)}=P(t, \varepsilon) .
\end{aligned}
$$

Thus $P$ has period $\omega$ in $t$. The other requirements of the theorem are obviously satisfied.

We can now prove without difficulty the main result of this section.

TheOREM 13. Let the Hermitian matrix $H(t, \varepsilon)$ be analytic in $\varepsilon$ at $\varepsilon=0$ and continuous in $t$ with period $\omega$. Moreover let the system

$$
J x^{\prime}=H(t, 0) x
$$

be strongly stable.

Then there exists a J-unitary matrix $S(t, \varepsilon)$ which is analytic in $\varepsilon$ at $\varepsilon=0$ and contimuously differentiable in $t$ with period $\omega$ such that the change of variables $x=S(t, \varepsilon) y$ transforms the perturbed system (13) into the autonomous system

$$
J y^{\prime}=F(\varepsilon) y,
$$

where $F(\varepsilon)$ is a real diagonal matrix analytic in $\varepsilon$ at $\varepsilon=0$. 
Let $X(t, \varepsilon)$ be the fundamental matrix of $(13)$ and let $P(t, \varepsilon)$ be the matrix appearing in the representation (19). The change of variables $x=P(t, \varepsilon) y$ transforms (13) into the autonomous system

$$
J y^{\prime}=K(\varepsilon) y .
$$

Moreover the monodromy matrix $e^{\omega J^{-1} K(0)}$ of the unperturbed system $J y^{\prime}=K(0) y$ is strongly stable. Therefore, by Theorem 7 and its Corollary, there exists a $J$-unitary matrix $G$ such that the change of variables $y=G z$ transforms $(20)$ into the system

$$
J z^{\prime}=G^{*} K(\varepsilon) G z,
$$

where $F=G^{*} K(0) G$ is a real diagonal matrix:

$$
F=\left[\lambda_{1}, \cdots, \lambda_{n}\right]
$$

and

$$
\lambda_{j}+\lambda_{k} \not \equiv 0(\bmod 2 \pi / \omega) \text { for } 1 \leqq j \leqq p<k \leqq n .
$$

The result now follows at once from Theorem 11.

The condition $\lambda_{j}+\lambda_{k} \neq 0$ appears to be stronger than the condition $\lambda_{j}+\lambda_{k} \neq 0$ which is required for the application of Theorem 11. However the matrix $A(0)=\omega J^{-1} K(0)$ in Lemma 6 has the property that distinct eigenvalues do not differ by multiples of $2 \pi i$ and so the two conditions are really equivalent.

\section{References}

[1] Chevalley, C., Theory of Lie groups, I, Princeton, 1946.

[2] Diliberto, S. P., On stability of linear mechanical systems, Proc. Internat. Sympos. Nonlinear Vibrations, Izdat. Akad. Nauk Ukrain. SSR, Kiev, 1963, Vol. 1, pp. $189-203$.

[3] Gelfand, I. M. and Lidskiǐ, V. B., On the structure of the domains of stability of linear canonical systems of differential equations with periodic coefficients (Russian), Uspehi Mat. Nauk (N.S.) 10 (1955) 3-40; Amer. Math. Soc. Transl. (2) 8 (1958) $143-181$.

[4] Jablonski, E., Théorie des permutations et des arrangements circulaires complets, J. Math. Pures Appl. (4) 8 (1892), 331-349.

[5] Krein, M. G., The basic propositions in the theory of $\lambda$-zones of stability of a canonical system of linear differential equations with periodic coefficients (Russian), Pamyati A. A. Andronova, Izdat. Akad. Nauk. SSSR, Moscow, 1955, pp. 413-498.

[6] Kreìn, M. G. and Yakubovič, V. A., Hamiltonian systems of linear differential equations with periodic coefficients (Russian), Proc. Internat. Sympos. Nonlinear Vibrations, Izdat. Akad. Nauk Ukrain. SSR, Kiev, 1963, Vol. 1, pp. 277-305.

[7] Moser, J., New aspects in the theory of stability of Hamiltonian systems, Comm. Pure Appl. Math. 11 (1958) 81-114.

[8] Riesz, F. and Sz.-Nagy, B., Functional Analysis, New York, 1955, Chap. XI. 
[9] Yakubovic, V. A., Critical frequencies of quasicanonical systems (Russian), Vestnik Leningrad Univ. 13 (1958) 35-63.

[10] Yakubovič, V. A., The small parameter method for canonical systems with periodic coefficients, J. Appl. Math. Mech. 23 (1959) 17-43.

The Australian National University,

Canberra. 